\title{
Cross-talk Between the Heart and Arteries in 65+ Adults
}

\author{
Tomasz H Wierzba ${ }^{1}$, Stanislaw Zajaczkowski ${ }^{1}$, Pawel Figarski ${ }^{1}$, Robert A Olek ${ }^{2}$, Artur Polinski ${ }^{3}$ \\ ${ }^{1}$ Department of Physiology, Medical University of Gdansk, Gdansk, Poland \\ ${ }^{2}$ Department of Bioenergetics and Nutrition, Gdansk University of Physical Education and Sport, \\ Gdansk, Poland \\ ${ }^{3}$ Biomedical Engineering Department, Faculty of Electronics Telecommunication and Informatics, \\ Gdansk University of Technology, Poland
}

\begin{abstract}
Regulatory synchronization between the heart and the arterial walls is essential for optimal blood delivery to tissues. We investigated functional coherence between heart rhythm and aortic wall compliance in 30 volunteers aged 65 - 74. ECG, carotid and iliac pulse-wave were recorded and digitized at $2 \mathrm{kHz}$. Carotid-femoral pulsewave transit time (cfTT) which reflects aortic compliance was assessed using the intersecting tangent algorithm at time-point of the maximal upstroke of the second derivative of the filtered pulse signal. Time-series of 256 heart cycles were used for analysis of heart rate variability (HRV) and cfTT variability analysis. Averaged power spectral density (aPSD) was estimated within selected frequency ranges by fast Fourier transform (FFT) approach. Magnitude squared coherence (MSC) between the both spectra was estimated. All volunteers exhibited variable temporal patterns of both HRV and CfTT variability. aPSD was reduced along with decreasing of time-window from 60 to 240 s. High coherent states between HRV and cfTT variability were observed as a short time prominent MSC peaks in almost all participants. Individual patterns of irregular MSC changes in time but not a generalized model of its fluctuations seem to reflect dynamic functional interaction between the heart and aortic compliance at an advanced age.
\end{abstract}

\section{Introduction}

Optimal adjustment of arterial impedance to ejective performance of heart provides low energetic cost of oxygen delivery to tissues and is protective from left ventricle overload and related complications [1, 2]. Underlying role of neurogenic cardiovascular regulation, including autonomic nervous system (ANS) oscillatory control, is still poorly understood. Heart rate variability (HRV) analysis is commonly used noninvasive method to assess ANS regulation of heart rhythm. Pulse wave velocity (PWV) in the large arteries reflects compliance of their walls. We aimed to investigate functional coherence between heart rhythm and aortic wall compliance in senescent apparently healthy volunteers.

\section{Material and methods}

Single chest-lead ECG (AdInstruments, Australia) and pulse wave (PW) were simultaneously recorded in resting conditions for $5-7 \mathrm{~min}$ in 30 volunteers (20 females, 10 men) aged from 65 to 74 . Exclusion criteria include chronic cardiovascular or respiratory problems, cancer, diabetes, renal or hepatic insufficiency and also chronic treatment with thyroxine, beta-blockers, ACE inhibitors or calcium channel blockers. Plethysmography sensors (MLT1020PPG, AdInstruments, Australia) were placed onto the left carotid artery and right iliac artery to assess PW. ECG and PW raw signals digitized at $2 \mathrm{kHz}$ were preprocessed, including artifact correction.

The R-wave was detected using Pan-Tompkins algorithm [3]. The characteristic points of PW signal were estimated using the intersecting tangent algorithm at timepoint of the maximal upstroke of the second derivative of the filtered pulse signal [4]. The time difference between characteristic points from carotid and femoral artery (carotid-femoral pulse wave transit time; cfTT) was calculated, and the individual distance between sensors (cfD) was measured. Pulse wave velocity (PWV), which is a major index of aortic compliance, was assessed by dividing cfTT by cfD.

Time-series of subsequent RR intervals (RRi) extracted from ECG, and from cfTT periodogram were obtained in 60, 120, 180 and 240 seconds windows. HRV and cfTT variability analysis was performed using Matlab software. Spectral components were obtained by fast Fourier transform (FFT) approach. Since RRi are not equal, thus at the time points corresponding to the subsequent $\mathrm{R}$ waves of the ECG signal, the spike with an amplitude equal to the length of the RR interval and the cfTT value were generated. Then both such generated signals were linearly 
interpolated and resampled with $2 \mathrm{~Hz}$ sampling frequency. Power spectral density function (PSD) of RRi (PSD_RRi) and cfTT (PSD_cfTT) was calculated within the range from 0 to $1 \mathrm{~Hz}$. PSD values were then averaged (aPSD) within the five arbitrary selected frequency ranges: I) 0.04 to $0,15 \mathrm{~Hz}$ representing low frequency component (LF) [5]; II) LF segment: 0,07 to $0,15 \mathrm{~Hz}$; two bands representing standard high frequency spectrum (HF): III) 0,15 to $0.3 \mathrm{~Hz}$, and IV) 0,3 to $0,5 \mathrm{~Hz}$; V) extended $\mathrm{HF}$ band: 0,3 to $0,7 \mathrm{~Hz}$, and also 0.04 to 0.7 spectrum representing the whole tested regulatory range.

....The magnitude squared coherence (MSC) was calculated using the formula:

$$
M S C_{x y}(\mathrm{f})=\frac{\left|P_{x y}(\mathrm{f})\right|^{2}}{P_{x x}(\mathrm{f}) P_{y y}(\mathrm{f})}
$$

were $P_{x x}(\mathrm{f})$ and $P_{y y}(\mathrm{f})$ are power spectral densities, and $P_{x y}(\mathrm{f})$ is the cross power spectral density, of signals $\mathrm{x}$ (RRi) and y (cfTT). The calculations were carried out using Matlab function with standard settings [6, 7]. MSC between RRi and cfTT was calculated for 60, 120, 180 and 240 seconds windows. Then the both signals were recurrently shifted by one sample (0.5 second) and MSC was recalculated. Since one signal may affect another one, MSC was calculated also for signals shifted in time. The time shift was vary from -3 to +3 seconds with resolution 0.5 second (i.e. -6 samples to +6 samples).

\section{Results and discussion}

Basic anthropometric characteristics and cfPWV in the examined group is shown in Table 1 . BMI above $30 \mathrm{~kg} / \mathrm{m}^{2}$, indicative for obesity, was found in $36.7 \%$ volunteers, whereas cfPWV exceeding normal range of $10 \mathrm{~m} / \mathrm{s}$ was revealed in $16.7 \%$ of volunteers.

Table 1. Basic characteristic of the participants. Data shown as mean \pm SD. Abbreviations are explained in text.

\begin{tabular}{lcc}
\hline & Mean value & Range \\
\hline Age (years) & $68.10 \pm 2.33$ & $65-74$ \\
Body weight $(\mathrm{kg})$ & $75.69 \pm 16.95$ & $45.20-106.60$ \\
BMI $\left(\mathrm{kg} / \mathrm{m}^{2}\right)$ & $28.35 \pm 5.13$ & $18.44-40.03$ \\
cfPWV $(\mathrm{m} / \mathrm{s})$ & $8.87 \pm 2.07$ & $5.15-14.65$ \\
\hline
\end{tabular}

All participants exhibited variable patterns of the HRV and cfTT variability in the all the tested time-windows. Averaged spectral powers: aPSD_RRi and aPSD_cfTT decreased along with an increase of time-window from 60 to $240 \mathrm{~s}$ (Table 2), while MSC reduction was smaller. In each tested time window the aPSD_RRi or aPSD_cfTT was higher in LF bands ( 0.04 to $0.15 \mathrm{~Hz}$ or 0.07 to $0.15 \mathrm{~Hz}$ ) compared to $\mathrm{HF}$ segments $(0.15$ to $0.3 \mathrm{~Hz}, 0.3$ to $0.5 \mathrm{~Hz}, 0.3$ to $0.7 \mathrm{~Hz}$ ). MSC varied individually (Figure 1). Table 2. Averaged power spectral density (aPSD) and magnitude square coherence (MSC) within the frequency range 0.04 to $0.7 \mathrm{~Hz}$ calculated in different time windows. Data shown as mean \pm SD. Abbreviations are explained in text.

\begin{tabular}{cccc}
\hline $\begin{array}{c}\text { Time } \\
\text { window }\end{array}$ & aPSD_RRi & aPSD_cfTT & MSC \\
\hline $60 \mathrm{~s}$ & $1.98 \pm 0.80$ & $0.18 \pm 0.04$ & $0.26 \pm 0.09$ \\
$120 \mathrm{~s}$ & $1.51 \pm 0.35$ & $0.13 \pm 0.02$ & $0.28 \pm 0.07$ \\
$180 \mathrm{~s}$ & $1.26 \pm 0.18$ & $0.10 \pm 0.01$ & $0.26 \pm 0.04$ \\
$240 \mathrm{~s}$ & $1.07 \pm 0.09$ & $0.09 \pm 0.01$ & $0.25 \pm 0.03$ \\
\hline
\end{tabular}

Percentage of $0.0078 \mathrm{~Hz}$ bands with MSC $>0.3$ or $>0.5$ was revealed in $30 \%$ and $6.67 \%$ of participants, respectively, within the frequency range between 0.04 and $0.7 \mathrm{~Hz}$. Within the narrower preselected bands, especially the frequency segment 0.07 to $0.15 \mathrm{~Hz}$, high coherent states were disclosed in 60\% (MSC > 0.3) and 13.33\% (MSC > 0.5) of volunteers.
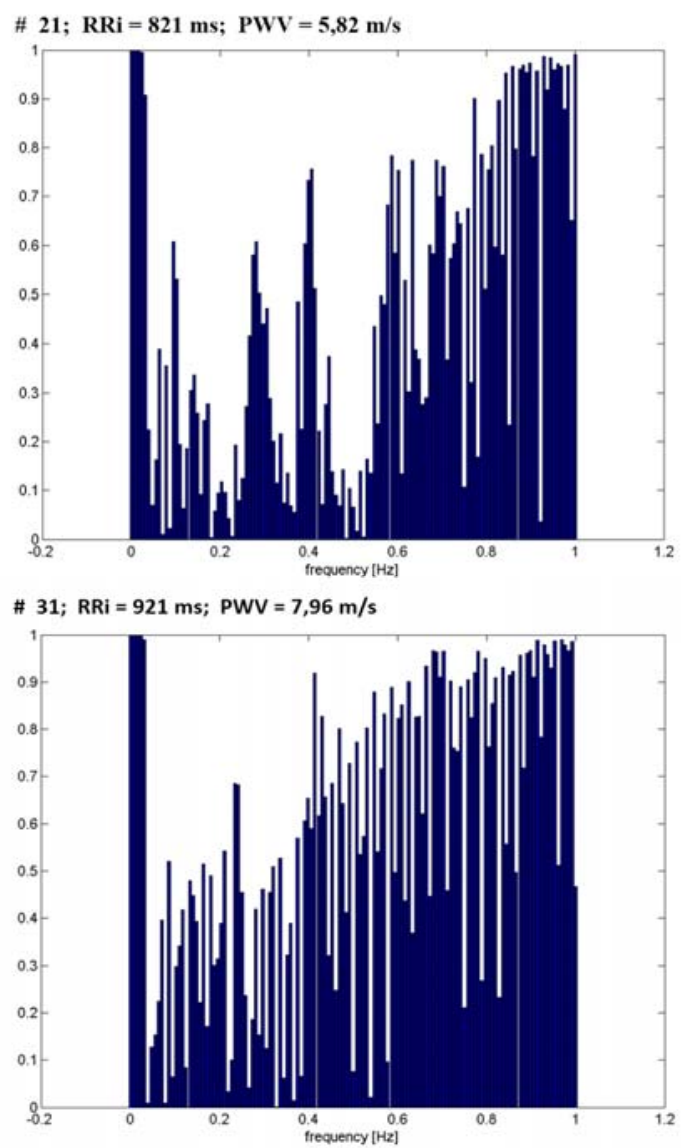

Figure 1. Magnitude squared coherence (MSC) of RRi and cfTT spectral powers - data from representative volunteers. Each bar denotes MSC within a $0.0078 \mathrm{~Hz}$ frequency range. Abbreviations are explained in text.

Proportion of MSC above 0.3 was higher in the RRi- and 
cfTT-power spectra calculated from $60 \mathrm{~s}$ or $120 \mathrm{~s}$ timewindows compared to those obtained from 180 and $240 \mathrm{~s}$ periods (Table 3$)$.

Table 3. Averaged power spectral density (aPSD) and magnitude square coherence (MSC) in selected frequency bands calculated in 120-s time window. Data shown as mean \pm SD. Abbreviations are explained in text.

\begin{tabular}{cccc}
\hline $\begin{array}{r}\text { Frequency } \\
\text { band (Hz) }\end{array}$ & aPSD_RRi & aPSD_cfTT & MSC \\
\hline $0.04-0.15$ & $3.23 \pm 1.51$ & $0.26 \pm 0.04$ & $0.31 \pm 0.11$ \\
$0.07-0.15$ & $3.16 \pm 0.75$ & $0.25 \pm 0.04$ & $0.32 \pm 0.13$ \\
$0.15-0.30$ & $2.38 \pm 0.64$ & $0.21 \pm 0.03$ & $0.29 \pm 0.12$ \\
$0.30-0.50$ & $1.61 \pm 0.49$ & $0.15 \pm 0.02$ & $0.26 \pm 0.09$ \\
$0.30-0.70$ & $1.21 \pm 0.36$ & $0.11 \pm 0.01$ & $0.25 \pm 0.09$ \\
\hline
\end{tabular}

a) Time-window $60 \mathrm{~s}$

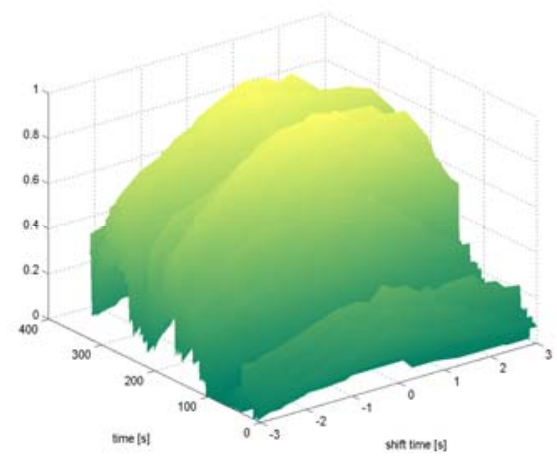

b) Time-window $120 \mathrm{~s}$

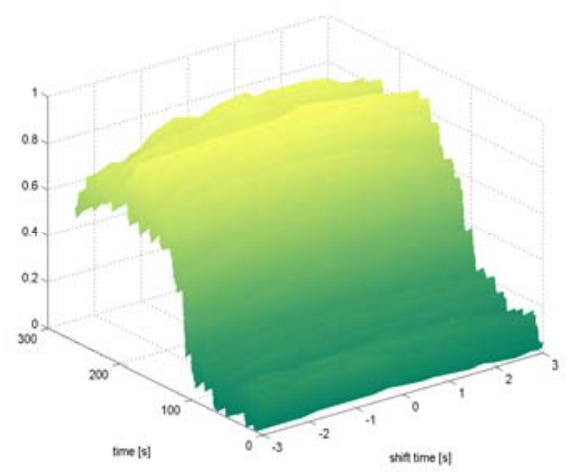

c) Time -window $180 \mathrm{~s}$

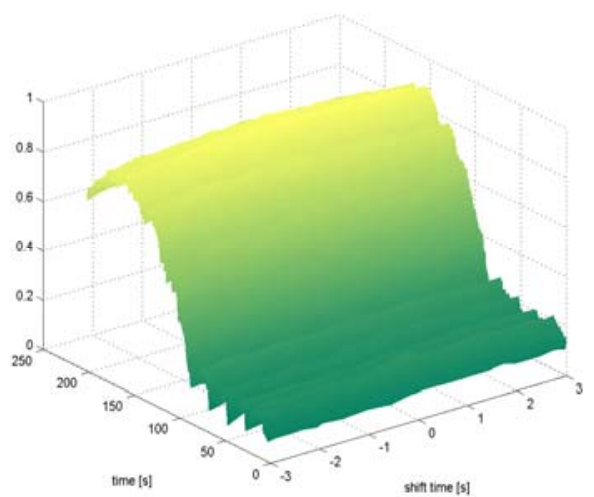

d) Time-window $240 \mathrm{~s}$

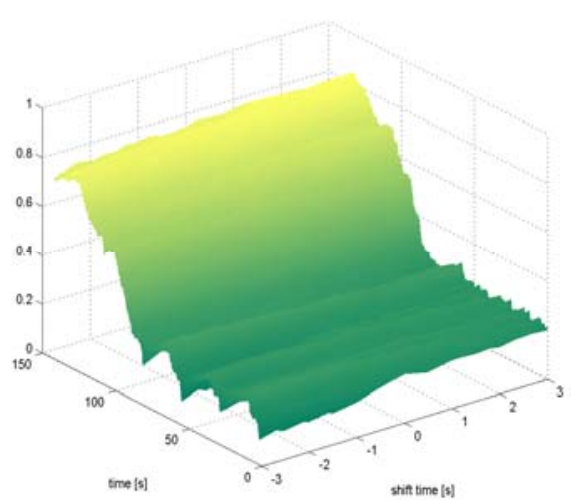

Figure 2 (a-d). Proportion of spectral lines for which MSC was greater than 0.5 with relation to number of all spectral lines in MSC (vertical axis) for calculation in timewindows of different lengths $(\mathrm{a}-\mathrm{d})$. MSC was calculated within a frequency range between 0.04 and $0.7 \mathrm{~Hz}$.

The MSC analysis was performed in subsequent timewindows of a given lengths with a time-shift of $0.5 \mathrm{~s}$. The beginning of the time axis (0) corresponds to the end of the first time-window of a given lengths. Shift-time axis reflects phase shift between HRV (PSD_RRi) and aortic transit time variability (PSD_cfTT). Zero value means that HRV and cfTT variability were compared at the same time. Abbreviations are explained in text

MSC varied in time. Different patterns of time-changes of MSC were observed in all individuals of the tested group. Time-window chosen for MSC estimation influenced its shape and amplitude (Figure 2). As shown in Table 2, calculations performed in wide time-windows provided the lowest variation and thus were chosen for further analyses performed in selected spectral ranges.

As shown in Figure 3a and $3 b$ exclusion of 0.04 to $0.07 \mathrm{~Hz}$ segment did not affect LF MSC. In turn, MSC patterns in extended $\mathrm{HF}$ ( 0.3 to $0.7 \mathrm{~Hz}$, Figure 3e) were definitely different from 0.15 to $0.3 \mathrm{~Hz}$ (Figure 3c) or 0.3 to $0.5 \mathrm{~Hz}$ segments (Figure 3d).

a) Frequency range: $0.04-0.15 \mathrm{~Hz}$ 


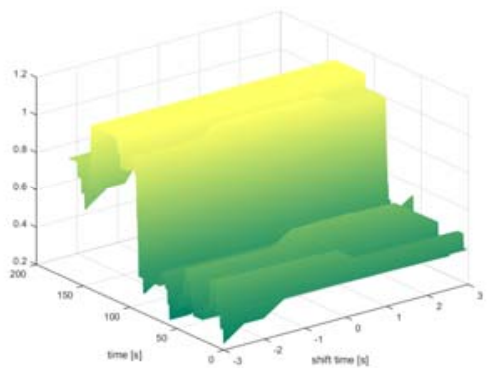

b) Frequency range: $0.07-0.15 \mathrm{~Hz}$

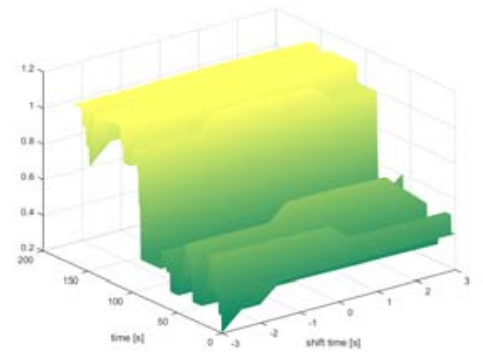

c) Frequency range: $0.15-0.3 \mathrm{~Hz}$

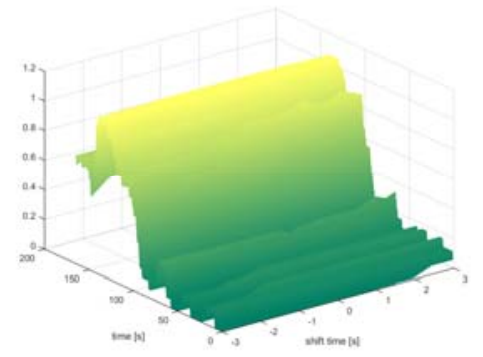

d) Frequency range $0.3-0.5 \mathrm{~Hz}$

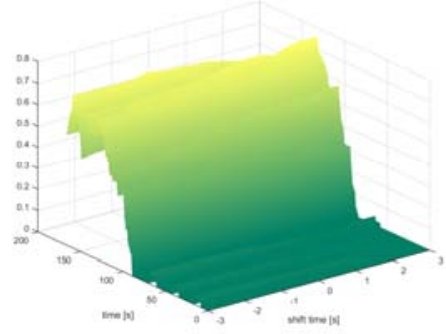

e) Frequency range $0.3-0.7 \mathrm{~Hz}$

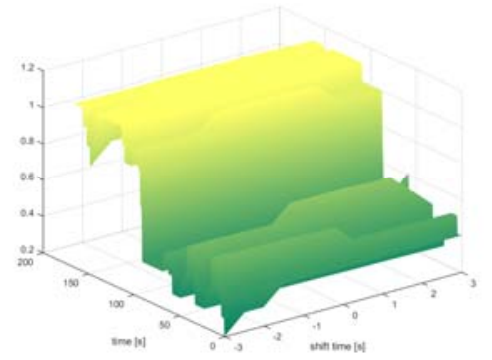

Figure 3 (a-e). Comparison of MSC of HRV (PSD_RRi) and aortic transit time variability (PSD_cfTT) calculated in different frequency bands in $240 \mathrm{~s}$ time-window. Vertical axis reflect proportion of spectral lines for which MSC was greater than 0.5 with relation to number of all spectral lines in MSC. Abbreviations are explained in text

\section{Summary}

Functional dynamic links between the heart rhythm and aortic compliance was confirmed with MSC analysis. Individual patterns of irregular temporal cardio-aortic synchronization but not a generalized model of its fluctuations seem to reflect dynamic functional interaction between the heart and aortic compliance at an advanced age.

\section{Acknowlegements}

The study was supported by National Science Center 2014/15/B/NZ7/00893 and by Medical University of Gdansk ST:02-0036/07/261

\section{References}

[1] H. T. Ma and Y. T. Zhang, "Spectral analysis of pulse transit time variability and its coherence with other cardiovascular variabilities," Conf Proc IEEE Eng Med Biol Soc, vol. 1, pp. 6442-6445, 2006.

[2] C. Lal et al., "Reduced baroreflex sensitivity, decreased heart rate variability with increased arterial stiffness in predialysis,” Indian J Nephrol, vol. 27, no. 6, pp. 446451, 2017.

[3] J. Pan and W. J. Tompkins, “A real-time QRS detection algorithm,” IEEE Trans Biomed Eng, vol. 32, no. 3, pp. 230-236, 1985.

[4] S. C. Millasseau, A. D. Stewart, S. J. Patel, S. R. Redwood, and P. J. Chowienczyk, "Evaluation of carotidfemoral pulse wave velocity: influence of timing algorithm and heart rate," Hypertension, vol. 45, no. 2, pp. 222-226, 2005.

[5] "Heart rate variability. Standards of measurement, physiological interpretation, and clinical use: Task Force of The European Society of Cardiology and The North American Society of Pacing and Electrophysiology,” Circulation, vol. 93, no. 5, pp. 1043-1065, 1996.

[6] L. Rabiner and B. Gold, Theory and application of digital signal processing. Englewood Cliffs, N. J.: Prentice Hall Inc., 1976.

[7] S. M. Kay, Modern spectral estimation: Theory and application. Englewood Cliffs: Prentice Hall, 1988.

Corresponding address:

Tomasz H. Wierzba, MD, PhD

Medical University of Gdansk

Department of Physiology

1 Debinki Street, 80-210 Gdansk, Poland

twierzba@gumed.edu.pl 